\title{
压电驱动六自由度指向平台的力电耦合分析*
}

\author{
刘 凡 ${ }^{1}$ 周进雄 $^{2}$ 马小飞 1 \\ (1. 西安空间无线电技术研究所 西安 710100 ;
}

2. 西安交通大学机械结构强度与振动国家重点实验室 西安 710049)

\begin{abstract}
摘要: 在高精度微调机构的研究中, 面临的是一个包括多个部件、刚性与柔性并存、综合的力学-电学问题, 对于机构的高精 度微调指向性能以及后续的控制研究中, 需要建立并得到整个系统的输入与输出的关系, 即压电驱动器的输入电压和微调机 构的指向关系。采用压电六自由度并联机构作为平台, 基于压电材料的力电耦合理论, 在考虑力电耦合效应、位移放大机构 的弹性变形的影响下, 提出并建立了平台的全系统解析和数值预测方法, 其中分别包括了整个平台系统的部件级和系统级的 分析理论及数值模拟方法。计算结果表明, 所提出的解析和数值方法能够对此类六自由度平台的运动进行预测, 通过压电驱 动器的输入电压得到最终的平台输出姿态, 这对高精度微调机构的设计提供了新的研究手段和重要指导依据。
\end{abstract}

关键词: 压电; 六自由度平台; 力电耦合; 弹性变形; 解析

中图分类号: TM282

\section{Electro-mechanical Coupling Analysis of Piezoelectrically Driven Six-degree-of-freedom Pointing Platform}

\author{
LIU Fan $^{1}$ ZHOU Jinxiong ${ }^{2}$ MA Xiaofei ${ }^{1}$ \\ (1. Xi'an Institute of Space Radio Technology, Xi' an 710100; \\ 2. State Key Laboratory for Strength and Vibration of Mechanical Structures and School of Aerospace, \\ Xi'an Jiaotong University, Xi'an 710049)
}

\begin{abstract}
In the research of high precision pointing mechanism, we are faced with a comprehensive mechanical-electrical problem including multiple components, rigidity and flexibility. For the high precision fine-tuning pointing performance of the mechanism and subsequent control research, it is necessary to establish and obtain the relationship between input and output of the entire system, namely the relationship between the voltage of piezoelectric actuator and the pointing of the mechanism. Based on the electromechanical coupling theory of piezoelectric materials, analytical and numerical prediction methods for the input voltage of piezoelectric actuator and the output attitude are established for a six-degree-of-freedom platform with considering the effects of electromechanical coupling, displacement amplification mechanism and elastic deformation of flexible components. It includes the component-level and system-level analysis theory and numerical simulation methods. The calculation results show that the proposed analytical and numerical methods can predict the motion of six-degree-of-freedom platform and provide important guidance for the platform design.
\end{abstract}

Key words: piezoelectric; six degree of freedom platform; electro-mechanical coupling; elastic deformation; analysis theory

\section{0 前言}

随着航天技术的发展, 卫星等航天器上的毫米 波、亚毫米波、太赫兹及光学有效载荷对指向机构 的要求越来越高 ${ }^{[1]}$ 。同时, 随着光学镜面的发展, 对光学镜面的高精度指向也提出了大量需求。所以,

* 国家自然科学基金重点资助项目(U1537213)。20190512 收到初稿, 20191015 收到修改稿
开展航天器六自由度指向微调系统的研究对于空间 航天器的发展具有重要的学术和工程意义 ${ }^{[2]}$ 。然而, 传统电机很难实现高效、高精度的驱动, 因此研发 具有更高能量密度、输出精度的六自由度指向微调 平台一直是各国学者的研究热点。此外, 柔性铰链 因为所具有的无摩擦、无间隙等的优点, 在高精度 机构中的应用也越来越广泛。西班牙 SENER 公司 等机构 ${ }^{[3-10]}$ 设计并研制了多种驱动类型的六自由度 指向平台。BLANCHARD ${ }^{[11]}$ 研究了一种应用在天文 望远镜的磁-弹簧驱动六自由度微调机构, 该机构动 
平台的坚向位移达到 $16 \mathrm{~mm}$, 分辨率达到 $20 \mu \mathrm{m}$ 。 MAHBOUBKHAH 等 ${ }^{[12]}$ 针对六自由度并联机构进 行了自由振动下的动态响应研究, 分别通过建立动 力学模型和有限元的方法对机构的振动特性进行了 计算和比较, 并分析了二者的差异。OLIVIER 等 ${ }^{[13]}$ 针对 NASA 的平流层气溶胶及气体试验 SAGE III 中的指向微调机构(HMA)的模态进行了研究, 包括 了从发射到运行时的三种主要姿态, 并考虑了夹持 部件的刚度的影响。AKDAG 等 ${ }^{[14]}$ 针对六自由度平 台的机电一体化问题进行了有限元的研究与分析, 并在六自由度平台的工作空间内进行了刚体动力 学、运动学和结构强度的分析, 并进行了试验测试 其驱动性能。然而, 在高精度六自由度指向平台的 研究中, 大多数学者是对平台振动模态、压电驱动 器的设计与仿真等方面单独进行了研究和分析, 并 且将机构各部分近似为刚体, 忽略其中柔性部件的 弹性变形, 例如柔性铰链等。因此缺少一个能够快 速、准确地预测平台的输入激励和输出姿态的综合 性的数学模型。本文设计了具有压电位移放大机构 的六自由度平台, 并建立了平台系统的输入电压与 输出姿态的解析表达式。同时, 本文还考虑了压电 驱动器中的力电耦合效应及柔性部件的弹性变形, 进一步揭示了具有位移放大机构的压电六自由度平 台的特性, 为高精度指向平台的设计与研究提供了 参考。

\section{1 压电堆建模与仿真}

\section{1 研究对象与研究方法}

研究对象是基于 COREMORROW 公司研发的 NAC2014-H62 型分立式压电陶瓷堆栈 ${ }^{[15]}$, 主要是由 低压压电陶瓷片堆叠形成压电陶瓷堆栈, 从而实现 较大的位移行程。在本文的研究中, 为了简化, 用 各向同性材料的等效压电陶瓷模型来对堆栈结构进 行等效计算, 如图 1 所示。

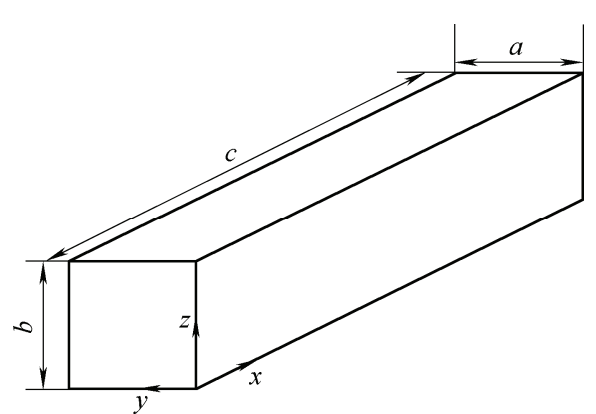

图 1 NAC2014-H62 型压电陶瓷堆栈模型示意图

如图 1 所示, 主要研究模型在 $z$ 方向施加电压 的条件下在 $x$ 方向产生位移。在本文中, 采用商用
有限元软件 ABAQUS 进行计算。ABAQUS 软件是 一个多场耦合分析的成熟 CAE 软件, 它提供了专 门的压电单元如 C3D8E, 可以进行精确的含压电 作动器的智能结构的建模与仿真。在 ABAQUS 中, 压电材料的本构方程使用 e-form 形式 ${ }^{[16]}$, 如 下所示

$$
\begin{gathered}
\sigma_{i j}=c_{i j k l}^{E} \varepsilon_{k l}-e_{k i j} E_{k} \\
D_{i}=e_{i k l} \varepsilon_{k l}+\lambda_{i k}^{\varepsilon} E_{k}
\end{gathered}
$$

式中, $c_{i j}$ 为压电材料的弹性常数; $\sigma_{i j}$ 表示应力分量; $\varepsilon_{k l}$ 表示应变分量; $D_{i}$ 表示电位移分量; $E_{k}$ 表示电 场强度; $\lambda_{i k}^{\varepsilon}$ 表示介电常数。

\section{2 结果与讨论}

压电陶瓷堆栈尺寸分别为: $a=b=7 \mathrm{~mm}$, $c=62 \mathrm{~mm}$, 由三维 8 节点线性压电实体单元 C3D8E 进行划分, 对其在施加 $150 \mathrm{~V}$ 电压的条件下的刚度 和位移分别进行了计算, 计算结果如图 2 所示, 并 将结果与试验值 ${ }^{[15]}$ 进行比较, 如表 1 所示。
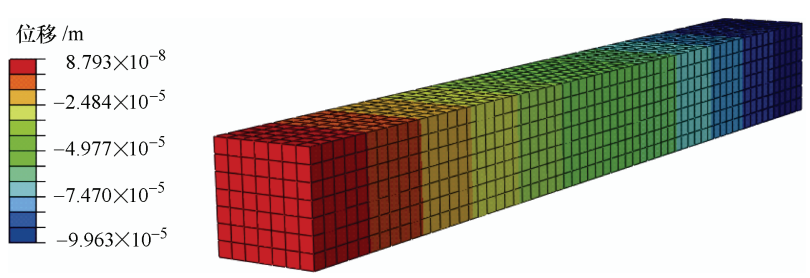

图 2 压电陶瓷堆栈有限元计算

表 1 计算结果

\begin{tabular}{cccc}
\hline 技术参数 & 试验值 & 计算值 & 相对误差 $(\%)$ \\
\hline 刚度 $/ \mathrm{N} / \mu \mathrm{m})$ & 20.0 & 21.6 & 8.0 \\
位移 $/ \mu \mathrm{m}$ & 99.0 & 99.7 & 2.1 \\
\hline
\end{tabular}

由表 1 可以看到, 所采用的等效计算能够较 好地预测实现结果, 因此利用有限元进行压电材 料的力电耦合建模与仿真能够描述其电致应变的 行为。

\section{2 压电堆驱动位移放大机构的建模与 仿真}

\section{1 研究对象与研究方法}

压电堆位移放大机构由 NAC2014-H62 型压电 堆和菱形位移放大机构组成, 放大机构材料采用 $65 \mathrm{Mn}$ 弹簧钢。同样, 在本节的研究中, 为了简化, 用各向同性材料的等效压电陶瓷模型来对堆栈结构 进行等效计算。压电堆和位移放大机构之间为刚性 连接。其中压电堆在电压的激励下在轴向方向产生 位移, 以此作为整个放大机构的位移激励, 其工作 
原理示意图如图 3 所示。

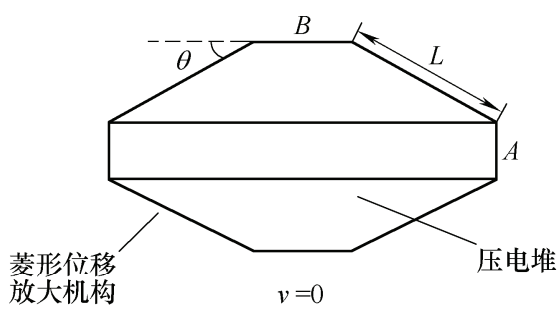

(a)

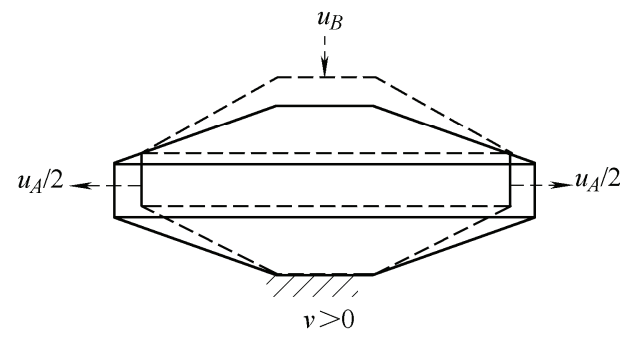

(b)

图 3 压电驱动放大机构示意图

图 3a 表示机构未施加电压的初始状态, 框架 顶部水平段和斜边所形成的角度为 $\theta$, 斜边长度为 $L$ 。图 $3 \mathrm{~b}$ 表示机构中的压电堆受到电压激励时整个 机构的状态。因为压电堆在轴向方向产生位移驱使 菱形框架产生变形, 使框架的 $A$ 点与 $B$ 点分别产 生位移。

由菱形位移放大机构的放大系数 $F^{[17]}$ 可得

$$
\begin{gathered}
F=\frac{u_{B}}{u_{A}}=\frac{c^{2} \tan \theta /\left(2 k_{r} \cos ^{2} \theta / k_{t}+c^{2} \tan ^{2} \theta\right)}{1+\frac{2}{c^{2} k_{p} \tan ^{2} \theta / 2 k_{r}+k_{p} \cos ^{2} \theta / k_{t}}} \\
c=L / 2 \\
k_{t}=E b t / L \\
k_{r}=E b t^{3} / 12 L
\end{gathered}
$$

式中, $k_{t} 、 k_{r}$ 分别为斜边梁的轴向刚度和转动刚度; $b 、 t$ 分别为斜边的厚度与宽度; $E$ 为菱形机构的弹 性模量。

当压电堆在自由状态下施加电压时, 由式(1) 可得

$$
\begin{gathered}
\varepsilon_{11}=\frac{M}{N} \cdot E_{3} \\
M=\left(e_{31} c_{13}-e_{33} c_{12}\right)\left(c_{13} c_{11}-c_{12} c_{13}\right)- \\
\left(c_{13} c_{13}-c_{12} c_{33}\right)\left(e_{31} c_{11}-e_{31} c_{12}\right) \\
N=\left(c_{11} c_{13}-c_{12} c_{13}\right)\left(c_{13} c_{11}-c_{12} c_{13}\right)- \\
\left(c_{11} c_{11}-c_{12} c_{12}\right)\left(c_{13} c_{13}-c_{12} c_{33}\right)
\end{gathered}
$$

式中, $\varepsilon_{11}$ 为 $x$ 方向应变; $E_{3}$ 为 $z$ 方向电场强度。由 此可得压电堆在自由状态下施加电压产生时轴向方 向产生的位移 $u_{p}$

$$
u_{p}=\frac{M L_{1}}{N L_{3}} U=\mathrm{d} U
$$

式中, $L_{1} 、 L_{3}$ 为压电堆 $x$ 方向和 $z$ 方向长度; $d$ 为 压电变形系数; $U$ 为 $z$ 方向所施加的电压。

当压电堆受到菱形框架的约束时, 可以得到施 加电压 $U$ 后压电堆轴向产生的位移 $u_{A}$

$$
\begin{gathered}
u_{A}=\frac{u_{p}}{1+k_{i n} / k_{p}} \\
k_{\text {in }}=\frac{2}{\frac{c^{2} \tan ^{2} \theta}{2 k_{r}}+\frac{\cos ^{2} \theta}{k_{t}}}
\end{gathered}
$$

式中, $k_{p}$ 为压电堆的刚度; $k_{i n}$ 为菱形框架的横向 刚度。

$$
u_{B}=\frac{c^{2} \tan \theta /\left(2 k_{r} \cos ^{2} \theta / k_{t}+c^{2} \tan ^{2} \theta\right)}{1+\frac{2}{c^{2} k_{p} \tan ^{2} \theta /\left(2 k_{r}\right)+k_{p} \cos ^{2} \theta / k_{t}}} \cdot \frac{\mathrm{d} U}{1+k_{i n} / k_{p}}
$$

由此, 通过式(6)建立了压电菱形位移放大机构 的输入电压 $U$ 与输出位移 $u_{B}$ 之间的解析式。

\section{2 结果与讨论}

压电堆驱动位移放大机构斜边长度 $L=24 \mathrm{~mm}$, 如图 4 所示, 斜边角度 $\theta=7.5^{\circ}, E=200 \mathrm{GPa}, b=7$ $\mathrm{mm}, t=2 \mathrm{~mm}$, 菱形框架的横向刚度 $k_{i n}=1 \times 10^{7} \mathrm{~N} / \mathrm{m}$, 压电堆的刚度 $k_{p}=2 \times 10^{7} \mathrm{~N} / \mathrm{m}, \mathrm{d}=6.65 \times 10^{-7} \mathrm{~m} / \mathrm{V}$ 。

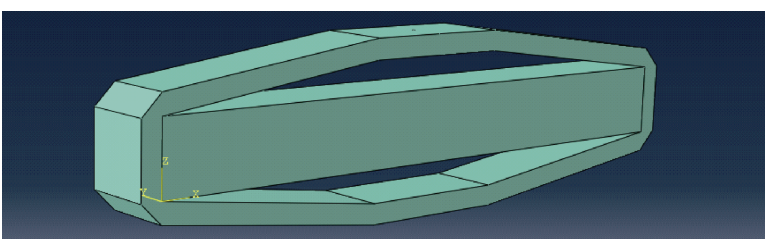

图 4 压电驱动位移放大机构

同样，压电堆由三维 8 节点线性压电实体单元 C3D8E 进行划分, 对其在施加 $150 \mathrm{~V}$ 电压的条件下 位移放大机构的位移进行了计算，并与式(6)的理论 值进行对比，计算结果如图 5 和表 2 所示。

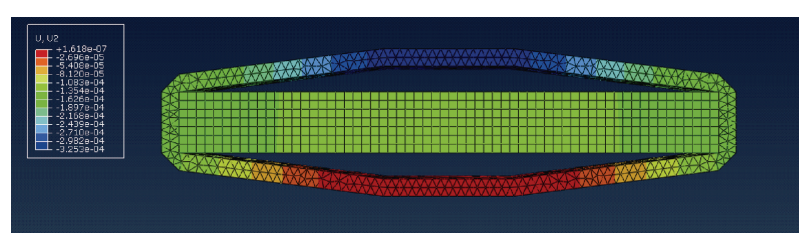

图 5 压电驱动位移放大机构计算

表 2 计算结果

\begin{tabular}{cccc}
\hline 参数 & 理论值 $/ \mathrm{mm}$ & 仿真值 $/ \mathrm{mm}$ & 相对误差 $(\%)$ \\
\hline$A$ 点位移 & $6.65 \times 10^{-2}$ & $6.60 \times 10^{-2}$ & 0.8 \\
$B$ 点位移 & 0.33 & 0.32 & 3.0 \\
放大系数 & 5.04 & 4.85 & 3.8 \\
\hline
\end{tabular}

由表 2 可以看到，本文所建立的解析表达式能够 
较好地预测仿真试验结果, 由此建立了压电驱动位移 放大机构的输入电压与输出姿态的解析表达式。

\section{3 六自由度平台的建模与仿真}

\section{1 研究对象}

六自由度平台基于高精度压电堆驱动方式, 采用 具有菱形位移放大机构和全柔性铰链的 Stewart 平台 结构, 用于实现六自由度的调节。该机构采用立方体 型的并联平台构型, 上下平台的面积、6 根支腿的长 度均相同, 具有惯量小、控制精度高等优点。平台总 体结构如图 6 所示, 该六自由度调节平台由位于上部 的位移输出平台、位于底部的固定底座和六个可伸缩 的菱形压电驱动器构成。其中位移输出平台用于平动 和指向调整，6根支腿和上下平台之间均采用基于柔 性铰链设计的高精度铰链连接, 并分别进行了编号。 该结构具有结构紧凑, 无磁性等特点。

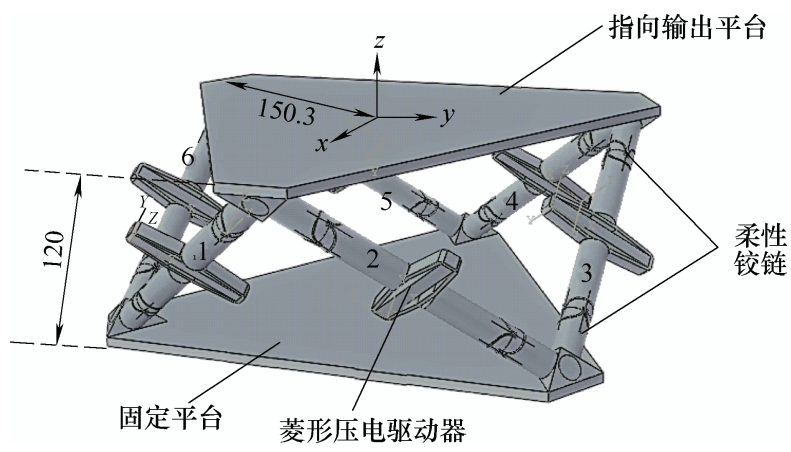

图 6 压电驱动六自由度平台结构图 $(\mathrm{mm})$

\section{2 运动学分析}

采用六自由度并联平台的矢量表示法来分析支 腿伸长量与上下平台的关系, 如图 7 所示, 并建立 固定坐标系 $\{B\}$ 和动坐标系 $\{P\}$ 。

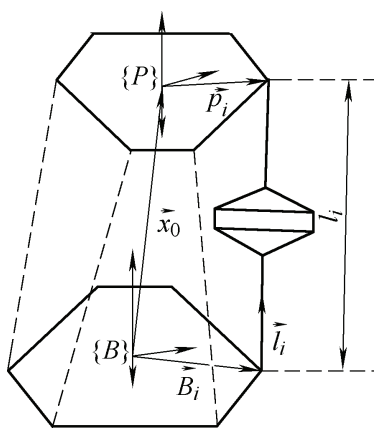

图 7 六自由度平台运动学模型

上平台在固定坐标系中有位置和姿态两个参 数, 位置是动平台中心的位置矢量 $\boldsymbol{x}_{0}$ 。设上平台绕 $x$ 轴旋转的角为 $\varphi$, 绕 $y$ 轴旋转的角为 $\theta$, 绕 $z$ 轴 旋转的角为 $\psi$, 则上平台的姿态表示为

$$
\boldsymbol{l}_{i}=\boldsymbol{p}_{i}^{B}+\boldsymbol{x}_{0}-\boldsymbol{B}_{i}=\boldsymbol{R} \cdot \boldsymbol{p}_{i}^{p}+\boldsymbol{x}_{0}-\boldsymbol{B}_{i} \quad i=1,2, \cdots, 6
$$

$$
\mathbf{R}=\mathbf{R}_{z}(\psi) \cdot \mathbf{R}_{y}(\theta) \cdot \mathbf{R}_{x}(\varphi)=
$$

$$
\left(\begin{array}{cc}
\cos \psi \cos \theta & \cos \psi \sin \theta \sin \varphi-\sin \psi \cos \varphi \\
\sin \psi \cos \theta & \cos \psi \cos \varphi+\sin \psi \sin \theta \sin \varphi \\
-\sin \theta & \cos \theta \sin \varphi \\
\sin \psi \sin \varphi+\cos \psi \sin \theta \cos \theta \\
\sin \psi \sin \theta \cos \varphi-\cos \psi \sin \varphi \\
\cos \theta \cos \varphi
\end{array}\right)
$$

式中, $\boldsymbol{R}$ 为从 $\{P\}$ 到 $\{B\}$ 的旋转矩阵, $\boldsymbol{l}_{i}=\left[l_{i x}, l_{i y}, l_{i z}\right]$ 为支腿在坐标系 $\{B\}$ 的矢量, $\boldsymbol{p}_{i}^{p}$ 为 $\boldsymbol{p}_{i}$ 在坐标系 $\{P\}$ 中的矢量, $\boldsymbol{p}_{i}^{B}$ 为 $\boldsymbol{p}_{i}$ 在坐标系 $\{B\}$ 中的矢量, $\boldsymbol{B}_{i}$ 为 下平台铰点在坐标系 $\{B\}$ 中的矢量。

压电驱动器的伸缩使支腿产生位移 $\Delta l_{i}$

$$
\Delta l_{i}=\sqrt{l_{i x}^{2}+l_{i y}^{2}+l_{i z}^{2}}-l_{0} \quad i=1,2, \cdots, 6
$$

式中, $l_{0}$ 为支腿的初始长度。

$$
\text { 由式(6) (9) 可得 }
$$

$$
\begin{gathered}
U_{i}=\frac{\left(\left|\boldsymbol{R} \cdot \boldsymbol{p}_{i}^{p}+\boldsymbol{x}_{0}-\boldsymbol{B}_{i}\right|-l_{0}\right) \cdot\left(1+k_{i n} / k_{p}\right)}{c^{2} \tan \theta /\left(2 k_{r} \cos ^{2} \theta / k_{t}+c^{2} \tan ^{2} \theta\right)} \\
\frac{1+\frac{2}{c^{2} k_{p} \tan ^{2} \theta / 2 k_{r}+k_{p} \cos ^{2} \theta / k_{t}}}{d} \\
i=1,2, \cdots, 6
\end{gathered}
$$

因此, 由式(10)可得到在上平台某一位姿 $[x, y$, $z, \varphi, \theta, \psi]$ 下, 每个压电驱动器所需要施加的电压 $U_{i}(i=1,2, \cdots, 6)$ 。

\section{3 柔性铰链设计}

与传统的铰链相比, 柔性铰链无摩擦, 无连接 间隙，无滞后，可大幅度提高精密位移调节的分辨 率。针对六自由度平台的高精度驱动方式, 采用柔 性铰链机构来连接上下平台和作动器, 如图 $8 \mathrm{a}$ 所 示, 由两个柔性铰链单元正交串联而成, 可以进行 两个正交方向自由度的转动。柔性铰链采用 $65 \mathrm{Mn}$ 弹簧钢, 各参数如图 $8 \mathrm{~b}$ 所示, 外径和内径分别为 $R=7.5 \mathrm{~mm}, r=3.75 \mathrm{~mm}, t=1 \mathrm{~mm}, a=b=7 \mathrm{~mm}$, 弹性模量 $E=200 \mathrm{GPa}$ 。

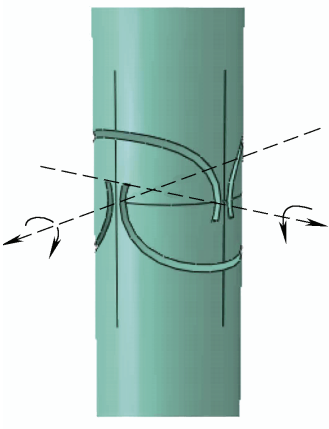

(a)

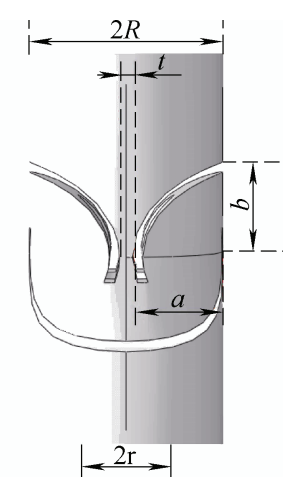

(b)
图 8 柔性铰链结构尺寸图 
利用有限元软件进行应力分析, 如图 9 所示, 当柔性铰链达到 $1^{\circ}$ 的极限弯曲变形时, 最大应力为 $163 \mathrm{MPa}$, 远小于弹簧钢 $65 \mathrm{Mn}$ 的许用应力 $\sigma=342$ $\mathrm{MPa}$ 。说明柔性铰链的结构安全可靠, 满足六自由 度平台的运动要求。


图 9 柔性铰链应力分析

\section{4 结果与讨论}

我们对压电驱动六自由度平台整体进行了有限 元力电耦合分析与计算, 并与式(10)的理论值进行 对比, 具体的求解步骤如下所示。

(1) 设置上平台初始位姿 $[x, y, z, \varphi, \theta, \psi]$ 。

(2) 通过式(10)得到在位姿 $[x, y, z, \varphi, \theta, \psi]$ 下的 解析解: 即每个支腿的位移 $\Delta l_{i}$ 和每个压电驱动器所 需施加的电压 $U_{i}(i=1,2, \cdots, 6)$ 。

(3) 建立平台的有限元模型, 将 $U_{i}$ 作为激励施 加到每个压电驱动器, 通过计算得到上平台的输出 位姿 $\left[x^{\prime}, y^{\prime}, z^{\prime}, \varphi^{\prime}, \theta^{\prime}, \psi^{\prime}\right]$ 。

(4) 将 $[x, y, z, \varphi, \theta, \psi]$ 与 $\left[x^{\prime}, y^{\prime}, z^{\prime}, \varphi^{\prime}, \theta^{\prime}, \psi^{\prime}\right]$ 进行 对比, 得出相对误差。

考虑到结构的对称性, 仅对上平台的单自由度 的平动和转动分别进行计算。图 10 表示对初始位姿 $[0,0,-0.5 \mathrm{~mm}, 0,0,0]$ 进行理论计算, 并将得到的电 压 $U_{i}$ 施加到有限元模型每个驱动器后得到输出位 姿。表 3、4 分别表示理论计算结果和有限元的仿真 计算值, 支腿序号如图 6 所示。



图 10 六自由度平台位移云图

表 3 平动状态下支腿位移的理论值

\begin{tabular}{ccccccc}
\hline 支腿序号 & 1 & 2 & 3 & 4 & 5 & 6 \\
\hline 支腿位移 $/ \mathrm{mm}$ & -0.2873 & -0.2883 & -0.2884 & -0.286 & -0.2876 & -0.2892 \\
电压 $/ \mathrm{V}$ & 162.8 & 163.4 & 163.4 & 162.2 & 163.0 & 163.9 \\
\hline
\end{tabular}

表 4 平动状态下位移的有限元仿真值

\begin{tabular}{cccc}
\hline & 初始值 & 仿真计算值 & 相对误差 $(\%)$ \\
\hline 位移 $/ \mathrm{mm}$ & -0.50 & -0.49 & 2.6 \\
\hline
\end{tabular}

图 11 表示对初始位姿 $\left[0,0,0,0,0.2^{\circ}, 0\right]$ 进行理 论计算, 表 5、6 分别表示理论值和有限元仿真计 算值。

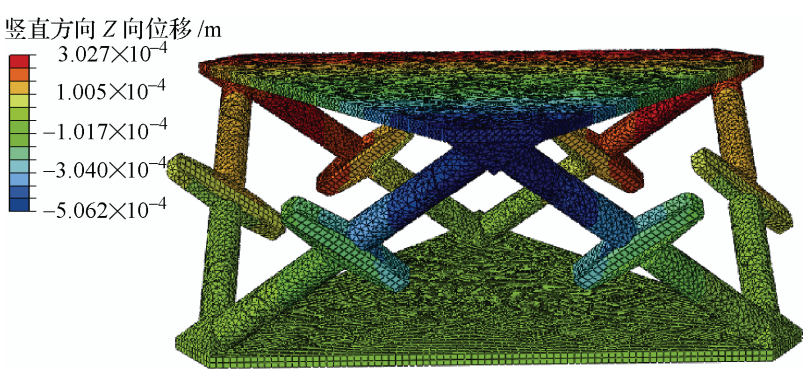

图 11 六自由度平台位移云图

表 5 转动状态下支腿位移的理论值

\begin{tabular}{ccccccc}
\hline 支腿序号 & 1 & 2 & 3 & 4 & 5 & 6 \\
\hline 支腿位移 $/ \mathrm{mm}$ & -0.2902 & -02912 & 0.1598 & 0.1334 & 0.1320 & 0.1590 \\
电压 $/ \mathrm{V}$ & 164.5 & 165.0 & -90.6 & -75.6 & -74.8 & -90.1 \\
\hline
\end{tabular}

表 6 转动状态下转动角的有限元仿真值

\begin{tabular}{cccc}
\hline & 初始值 $/\left(^{\circ}\right)$ & 仿真计算值 $/\left(^{\circ}\right)$ & 相对误差 $(\%)$ \\
\hline 转动角 & 0.20 & 0.19 & 2.1 \\
\hline
\end{tabular}

由表 4 和表 6 可以看到, 所建立的压电驱动六 自由度平台的解析表达式能够较好地预测仿真试验 结果, 由此, 建立了关于平台系统的输入电压与输 出姿态的解析表达式, 并在其中还考虑了压电驱动 器中的力电耦合效应及柔性部件的弹性变形。

\section{4 结论}

（1）本文基于压电材料的本构方程，分析了压 电材料的力电耦合行为, 并通过有限元仿真进行计 算, 揭示了压电材料的力电耦合行为特性与机理。

(2) 建立了压电驱动位移放大机构的输入电压 与输出位移的解析表达式, 并通过有限元仿真计算 进行验证。结果表明, 解析表达式能够准确地预测 仿真试验的结果。

(3) 提出并建立了压电驱动六自由度平台的全 系统解析表达式和仿真模型, 其中考虑了压电材料 的力电耦合效应、位移放大机构的放大效应和柔性 部件的弹性变形, 通过分析压电作动器的输入电压 对平台姿态的影响并进行理论和仿真计算, 说明所 提出的解析表达式能够准确地对平台的运动进行预 测。本文提出的解析表达式适用于含有压电材料和 弹性变形的平台, 形式简单, 能够直观、准确地对 
此类构型的六自由度平台进行预测, 研究结果对高 精度指向机构的设计与研究提供了指导意义和参考 价值。

\section{参 考 文 献}

[1] 杨建中. Stewart 并联机器人在航天器上的应用 $[C] / /$ 全 国第十二届空间及运动体控制技术学术会议论文集, 2006 年 8 月, 桂林. 北京: 中国自动化学会, 2007: 426-431.

YANG Jianzhong. Application of Stewart parallel robot on spacecraft $[\mathrm{C}] / /$ Proceedings of the 12th National Conf erence on Space and Sports Control Technology, August, 2006 , Guilin. Beijing : Chinese Association of Automation, 2007: 426-431

[2] 王兵. 指向、隔振一体化并联平台设计与优化 $[\mathrm{D}]$. 长 春: 中国科学院长春光学精密机械与物理研究所, 2016. WANG Bing. Optimization and design of a parallel platform combined vibration-isolation with pointing[D]. Changchun : Changchun Institute of Optics, Fine Mechanics and Physics, Chinese Academy of Science, 2016.

[3] DOMINGO M, VÁZQUEZ J. Large deployable antenna reflector trimming mechanism (LDA-RTM) $[\mathrm{C}] / /$ Proceedings of the 10th European Space Mechanisms and Tribology Symposium, September 24-26, San Sebastian, Spin. Noordwijk, Netherlands : ESA Publications Division, 2003: 191-198.

[4] 从强, 罗敏, 李伟杰. 空间机构技术发展趋势及展望 [J]. 载人航天, 2016, 22 (1): 1-8.

CONG Qiang, LUO Min, LI Weijie. Development trends and prospect of space mechanism[J]. Manned Spaceflight, 2016, 22(1): 1-8.

[5] YASUHIRO M, NANAKO M, HIROFUMI S, et al. The next generation space VLBI project, VSOP-2[J]. Proceedings of the International Astronomical Union, 2008, 3(S242): 517-521.

[6] 崔赪旻, 王典军. 跟踪与数据中继卫星星间链路天线驱 动机构技术综述 $[\mathrm{J}]$. 空间控制技术与应用, 2010,36(5): 32-37.

CUI Chengmin, WANG Dianjun. Survey of gimbal drive assembly mechanism of inter-orbit link antenna for tracking and data relay satellites[J]. Aerospace Control and Application, 2010, 36(5): 32-37.

[7] HAGIWARA Y, FOMALONT E, TSUBOI $\mathrm{M}$, et al. Approaching micro-arcsecond resolution with VSOP-2: Astrophysics and technologies[C]// Astrophysics and Technologies ASP Conference Series, December 3-7, 2007, Sagamihara, Kanagawa, Japan. San Francisco:
ASP, 2009: 495

[8] SNEED R C, CASH M F, CHAMBERS T S, et al. Six degree of freedom, sub-micrometer positioning system for secondary mirrors $[\mathrm{J}]$. Proceeding of SPIE, 2010, 7733(77332R): 1-11.

[9] CASH MF, ANDERSON EH, SNEED R, et al. Precision pointing parallel manipulator design for asymmetric geometries and cryogenic vacuum environments[J]. American society for Precision Engineering Annual Meeting, 2005, 1: 1 .

[10] ZIERER J J, MOCK J R, BENO J H, et al. The Development of high-precision hexapod actuators for the Hobby-Eberly telescope wide field upgrade[J]. Proceeding of SPIE, 2010, 7733(77331H): 1-12.

[11] BLANCHARD L. A tape-spring hexapod for deployable telescopes : Dynamics[C]// Robotics and Computer Integrated Manufacturing. Proc. Internet. Conf. on Space Optics, 2006, 10567(1056718): 1-6.

[12] MAHBOUBKHAH M, NATEGH M J, KHADEM S E. A comprehensive study on the free vibration of machine tools hexapod table[J]. International Journal of Advanced Manufacturing Technology, 2009, 40(11-12): 1239-1251.

[13] OLIVIER M, DUCHINI G, FLEMING P, Hexapod mechanical assembly modal survey test[J]. Proceedings of the 5th International Symposium on Environmental Testing for Space Programmes, 2004, SP-558: 165-172.

[14] AKDAG M, KARAgulle H, MALGACA L, An integrated approach for simulation of mechatronic systems applied to a hexapod robot[J]. Mathematics \& Computers in Simulation, 2012, 82(5): 818-835.

[15] Harbin Core Tomorrow Science \& Technology Co., Ltd[EB/OL]. [2018-12-05]. http: //www. coremorrow. com/proshow-9-167-1. html.

[16] 马尧. 基于 ABAQUS 的压电悬臂梁有限元仿真分析 [J]. 吉林化工学院学报, 2014, 31(7): 49-52.

MA Yao. Finite element analysis of piezoelectric cantilever beam based on ABAQUS[J]. Journal of Jilin Institute of Chemical Technology, 2014, 31(7): 49-52.

[17] SHAO Shubao, XU Minglong, ZHANG Shuwen, et al. Stroke maximizing and high efficient hysteresis hybrid modeling for a rhombic piezoelectric actuator[J]. Mechanical Systems and Signal Processing, 2016, 75(15): 631-647.

作者简介: 刘凡, 男, 1988 年出生, 博士。主要研究方向为结构设计与 优化。

E-mail: kinter616@163.com

马小飞(通信作者), 男, 1980 年出生, 博士, 研究员, 博士研究生导师。 主要研究方向为大型可展开空间天线结构。

E-mail: maxf041600@sina.com 